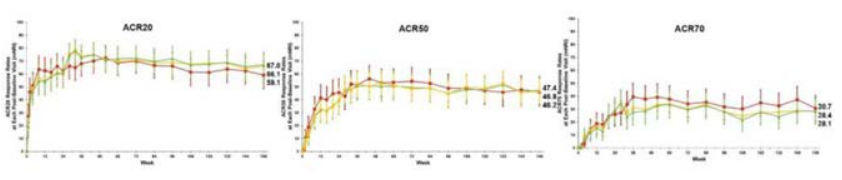

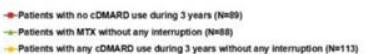

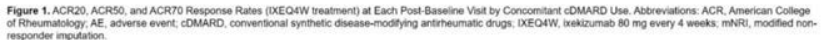

Disclosure of Interests: Laura C Coates: None declared, Andris Kronbergs Shareholder of: Eli Lilly and Company, Employee of: Eli Lilly and Company, Aubrey Trevelin Sprabery Shareholder of: Eli Lilly and Company, Employee of: Eli Lilly and Company, So Young Park Shareholder of: Eli Lilly and Company, Employee of: Eli Lilly and Company, Bernard Combe Grant/research support from: Novartis, Pfizer, Roche-Chugai, Consultant of: AbbVie; Gilead Sciences, Inc.; Janssen; Eli Lilly and Company; Pfizer; Roche-Chugai; Sanofi, Speakers bureau: Bristol-Myers Squibb; Gilead Sciences, Inc.; Eli Lilly and Company; Merck Sharp \& Dohme; Pfizer; Roche-Chugai; UCB, Atul Deodhar Grant/research support from: AbbVie, Eli Lilly, GSK, Novartis, Pfizer, UCB, Consultant of: AbbVie, Amgen, Boehringer Ingelheim, Bristol Myer Squibb (BMS), Eli Lilly, GSK, Janssen, Novartis, Pfizer, UCB, Speakers bureau: AbbVie, Amgen, Boehringer Ingelheim, Bristol Myer Squibb (BMS), Eli Lilly, GSK, Janssen, Novartis, Pfizer, UCB DOI: 10.1136/annrheumdis-2020-eular.3994

\section{SAT0411 EFFICACY AND SAFETY OF IXEKIZUMAB IN PATIENTS WITH PSORIATIC ARTHRITIS AND INADEQUATE RESPONSE TO TNF INHIBITORS: THREE YEAR RESULTS FROM A PHASE 3 STUDY (SPIRIT-P2)}

J. Gratacos-Masmitja ${ }^{1}$, A. Turkiewicz ${ }^{2}$, E. Dokoupilova ${ }^{3,4}$, A. M. Gellett ${ }^{5}$, A. T. Sprabery ${ }^{5}$, V. J. Geneus ${ }^{5}$, A. Constantin ${ }^{6} .{ }^{1}$ University Hospital Parc Tauli Sabadell, Barcelona, Spain; ${ }^{2}$ Rheumatology Associates PC, Birmingham, United States of America; ${ }^{3}$ Medical Plus s.r.o, Uherske Hradiste, Czech Republic; ${ }^{4}$ University of Veterinary and Pharmaceutical Sciences, Faculty of Pharmacy, Department of Pharmaceutics, Brno, Czech Republic; ${ }^{5}$ Eli Lilly and Company, Indianapolis, United States of America; ${ }^{6}$ Hospital Pierre Paul Riquet, Toulouse, France

Background: Ixekizumab (IXE) is a high affinity monoclonal antibody that selectively targets interleukin-17A. In the SPIRIT-P2 study, IXE every 4 (Q4W) or 2 $(\mathrm{Q} 2 \mathrm{~W})$ weeks was superior to placebo (PBO) in improving the signs and symptoms of psoriatic arthritis (PsA) at Week 24 in patients (pts) with prior inadequate response or intolerance to 1 or 2 tumor necrosis factor inhibitors (TNFi).

Objectives: To determine efficacy and safety of IXE treatment up to 3 years in pts with PsA.

Methods: In SPIRIT-P2 (NCT02349295), 310 pts entered the extension period where pts maintained their original ixekizumab dose, and placebo pts received IXEQ4W or IXEQ2W (1:1). Pts failing to demonstrate $\geq 20 \%$ improvement in both tender and swollen joint counts at Week 32, or any subsequent visit, were discontinued (mandatory discontinuation criteria). Efficacy outcomes were ACR20/50/70 response, Psoriasis Area and Severity Index (PASI) 75/90/100 response, Leeds Enthesitis Index (LEI), Leeds Dactylitis Index-Basic (LDIB), Minimal Disease Activity (MDA), and Disease Activity in Psoriatic Arthritis (DAPSA). Ad-hoc efficacy data are presented for intent-to-treat (ITT) pts initially randomized to IXE at Week 0 . Observed and modified non-responder imputation (mNRI; missing data treated as non-response for pts discontinued due to lack of efficacy or adverse events [AEs]) was applied to categorical measures. Observed and modified baseline observation carried forward (mBOCF) was applied to continuous efficacy measures. Safety was analysed in pts exposed to at least one dose of IXE.

Results: Of the 245 pts initially randomized to IXE at Week 0 (ITT), 64 (26.1\%) pts discontinued due to lack of efficacy and $22(9.0 \%)$ pts due to mandatory discontinuation criteria. Efficacy results are summarized below (Figure 1). Pts in SPIRIT-P2 who received IXEQ4W and IXEQ2W for 156 weeks reported sustained improvement in ACR responses and manifestations of PsA, including enthesitis, dactylitis, and skin outcomes. Treat-to-target measures such as MDA and DAPSA (Low Disease Activity or Remission) were achieved by $30.8 \%$ and $47.7 \%$ of pts, respectively on IXEQ4W, and by $29.2 \%$ and $40.7 \%$ of pts, respectively on IXEQ2W. Incidence rates (IR) of treatment-emergent adverse events (TEAEs) are provided below (Figure 2). Most TEAEs were mild or moderate in severity, and 38 out of 337 (5.9\%) pts (safety population) discontinued due to AEs. The most common TEAEs were infections $(I R=33.1)$ and injection site reactions $(\mathrm{IR}=5.4)$. Three deaths were reported in the study.

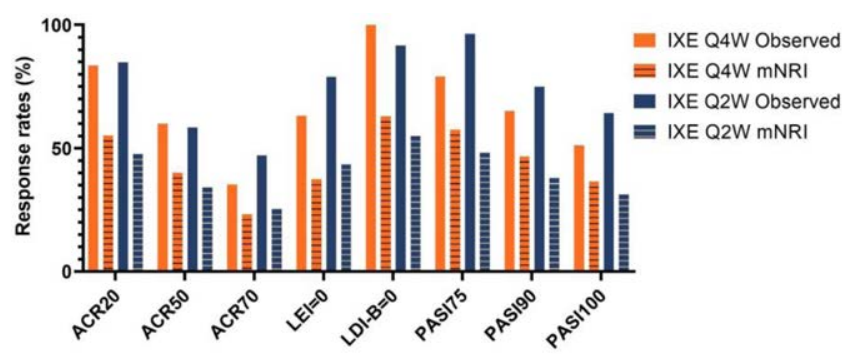

Figure 1. Efficacy Outcome Measures at Week 156 (Intent-to-treat Population) ACR=American College of Rheumatology; IXE=ixekizumab; LEI=Leeds Enthesitis Index; LDI$\mathrm{B}=$ Leeds Dactylitis Index-Basic; $\mathrm{mNRI}=$ modified non-responder imputation; PASI=Psoriasis Area and Severity Index; Q2W=every two weeks; Q4W=every four weeks.

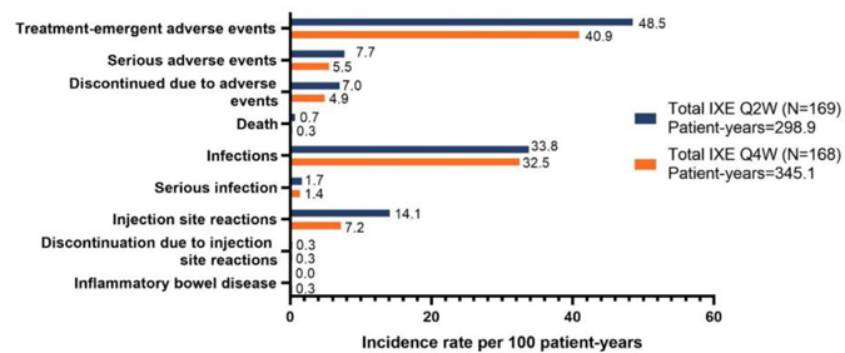

Figure 2. Safety Outcome Measures (Weeks 0-156). Safety was analysed in patients exposed to at least one dose of ixekizumab. During the double-blind treatment period (Weeks 0-24), one patient reported serious adverse events of anal fistula and anal abscess, which were considered by the sponsor to be IBD; however, an independent adjudication committee of external experts reviewed the case and determined the events to be "Not IBD."

Conclusion: In pts treated with IXE who had prior inadequate response or intolerance to 1 or $2 \mathrm{TNFi}$, improvements in the signs and symptoms of PsA persisted up to 3 years. No unexpected safety signals were observed, and the safety profile was consistent with previous studies of IXE.

Disclosure of Interests: Jordi Gratacos-Masmitja Grant/research support from: a grant from Pfizzer to study implementation of multidisciplinary units to manage PSA in SPAIN, Consultant of: Pfizzer, MSD, ABBVIE, Janssen, Amgen, BMS, Novartis, Lilly, Speakers bureau: Pfizzer, MSD, ABBVIE, Janssen, Amgen, BMS, Novartis, Lilly, Anthony Turkiewicz Grant/research support from: Received research grants from AbbVie, Eli Lilly and Company, Janssen, Novartis, and Pfizer, Consultant of: Received consulting fees from AbbVie, Eli Lilly and Company, Janssen, Novartis, and Pfizer, Speakers bureau: On speaker bureau for Abbvie, Eli Lilly and Company, Janssen, Novartis, Pfizer, Eva Dokoupilova Grant/research support from: Eli Lilly, AbbVie, Novartis, Amanda M. Gellett Shareholder of: Eli Lilly and company, Employee of: Eli Lilly and company, Aubrey Trevelin Sprabery Shareholder of: Eli Lilly and Company, Employee of: Eli Lilly and Company, Vladimir J. Geneus Shareholder of: Eli Lilly and Company, Employee of: Eli Lilly and Company, Arnaud Constantin Grant/research support from: Study was sponsored by Sanofi Genzyme, Consultant of: Consulting fees from Abbvie, BMS, Celgene, Gilead, Janssen, Lilly, Novartis, Pfizer, Roche, Sanofi, UCB

DOI: 10.1136/annrheumdis-2020-eular.1482

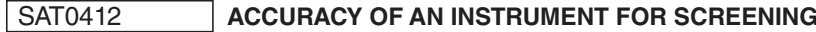 PSORIATIC ARTHRITIS AMONG PSORIATIC PATIENTS: RESULTS FROM THE MULTICENTRE ITALIAN STUDY HERACLES (SCREENING STRATEGIES FOR RHEUMATOLOGICAL REFERRAL OF PSORIATIC SUBJECTS AIMED TO DISCLOSE PSORIATIC ARTHRITIS)}

G. De Marco ${ }^{1}$, M. Manara ${ }^{2}$, P. Gisondi ${ }^{3}$, L. Idolazzi ${ }^{4}$, R. Ramonda ${ }^{5}$, S. Piaserico ${ }^{6}$, A. Cauli ${ }^{7}$, M. A. Cimmino ${ }^{8}$, V. Tomatis $^{8}$, C. Salvarani ${ }^{9}$, R. Scrivo ${ }^{10}$, A. Zanetti ${ }^{11}$, G. Carrara ${ }^{11}$, C. A. Scirè ${ }^{11}$, A. Cattaneo ${ }^{12}$, A. Marchesoni ${ }^{2} .{ }^{1}$ NIHR Leeds Biomedical Research Centre, Leeds Teaching Hospitals NHS Trust, Leeds Institute of Rheumatic and Musculoskeletal Medicine, University of Leeds, Leeds, United Kingdom; ${ }^{2}$ ASST Gaetano Pini-CTO, Milano, Italy; ${ }^{3}$ University of Verona, Dermatology and Venereology Section, Verona, Italy; ${ }^{4}$ University of Verona, Rheumatology Unit, Department of Medicine, Verona, Italy; ${ }^{5}$ University of Padova, Rheumatology Unit, Department of Medicine-DIMED, Padova, Italy; ${ }^{6}$ University of Padova, Dermatology Unit, Department of Medicine-DIMED, Padova, Italy; ${ }^{7}$ Università 
degli Studi di Cagliari, Cagliari, Italy; ${ }^{8}$ Università degli Studi di Genova, Genova, Italy; ${ }^{9}$ Università di Modena e Reggio Emilia, Reggio Emilia, Italy; ${ }^{10}$ Università La Sapienza, Roma, Italy; ${ }^{11}$ Italian Society for Rheumatology, Milano, Italy; ${ }^{12}$ IRCCS Ca' Granda Ospedale Maggiore Policlinico, UOC Dermatologia, Milano, Italy

Background: Identifying psoriatic arthritis (PsA) among people with psoriasis is often challenging due to low specificity of symptoms at early PsA stage and/ or delayed referral to the rheumatologist. Screening instruments -assisting the dermatologist to decide when rheumatological assessment is beneficial- have potential to reduce the diagnostic delay.

Objectives: To evaluate the accuracy of a dermatologist-filled-out questionnaire designed for screening PsA among psoriatic patients under dermatology care.

Methods: HERACLES is a multicentre, cross-sectional study running at 9 Italian dermatology and rheumatology tertiary centres. All participants were under dermatology care for skin psoriasis. Previous diagnosis of PsA precluded eligibility. Dermatologists at each site assessed consecutive psoriatic subjects, filled in the specifically-designed HERACLES questionnaire (HQ, Figure 1) and finally referred the participants to rheumatologists for clinical evaluation. All participants filled in the ToPAS, PASE, PEST and EARP questionnaires. Rheumatologists assessed the participants regardless of the questionnaires' scores. The gold standard applied to assess the instruments' accuracy was the diagnosis of PsA as established by the rheumatologists. ROC curve analysis evaluated the performance of the scores associated with the clinical criteria listed in the $H Q$, estimating the sensitivity and specificity of different cut-off levels. Further exploratory ROC curve analysis compared $\mathrm{HQ}$ performance to that of the other four questionnaires tested.

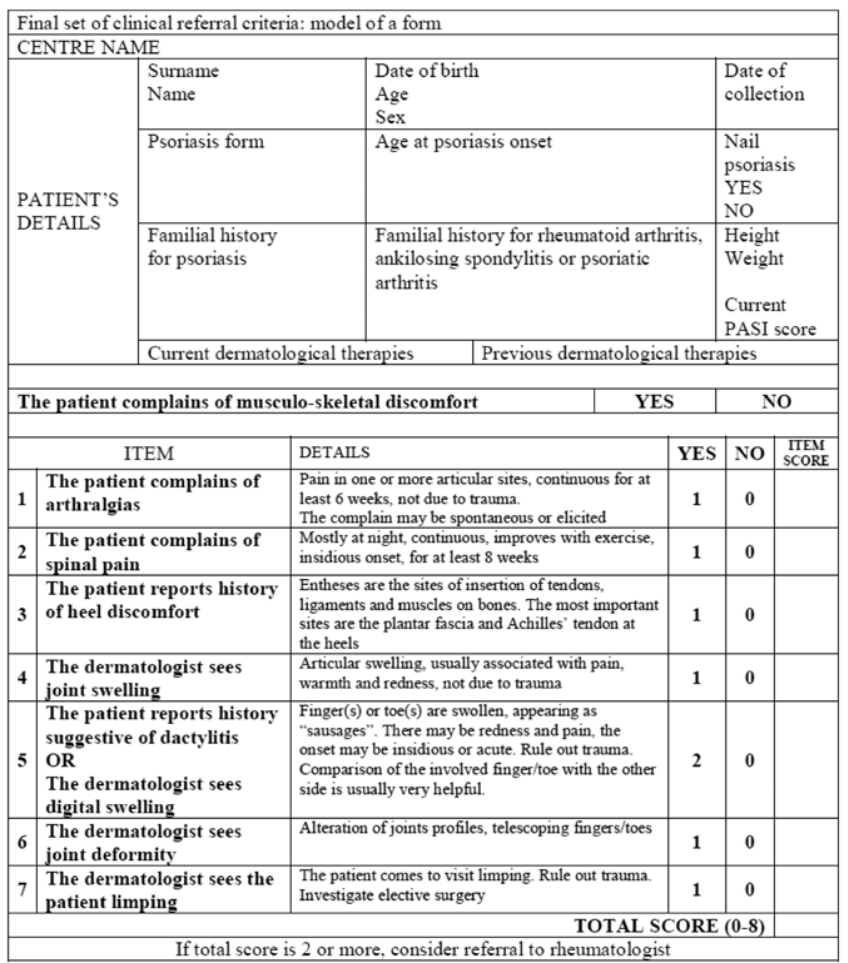

Figure 1.

Results: Out of 759 subjects enrolled, $524(69 \%)$ attended rheumatology assessment. Rheumatologists diagnosed PsA in 73/524 patients (13.9\%, Figure 2). Mean age was 53 (SD 16) years and $46 \%$ were female. Mean psoriasis duration was 20 (SD 19) years. The area under the ROC curve of HQ was 0.775 . The HQ score cut-off value of 2 yielded a sensitivity of $92 \%$ and a specificity of $47 \%$; a cut-off value of 3 yielded a sensitivity of $66 \%$ and a specificity of $75 \%$. The comparison between the ROC curve of the $\mathrm{HQ}$ and those of the other four questionnaires evaluated did not show any significant difference $(p=0.523$ versus TOPAS; $p=0.201$ versus PASE; $p=0.345$ versus PEST and $p=0.240$ versus EARP).
Flow Chart, HERACLES study

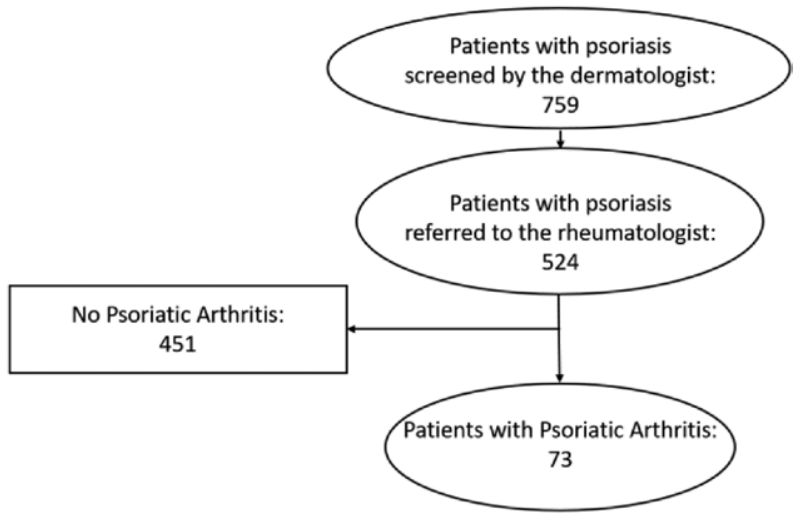

Figure 2.

Conclusion: The HERACLES questionnaire, a tool designed for dermatologists, showed good sensitivity and specificity in identifying PsA cases among subjects with cutaneous psoriasis.

Acknowledgments: The HERACLES project was supported by a research grant from FIRA and Pfizer Italia SRL

Disclosure of Interests: Gabriele De Marco: None declared, Maria Manara Consultant of: Consultant and/or speaker for Eli-Lilly, MSD, Sanofi-Genzyme, Novartis, Alfa Wasserman and Cellgene, Speakers bureau: Consultant and/ or speaker for Eli-Lilly, MSD, Sanofi-Genzyme, Novartis, Alfa Wasserman and Cellgene, Paolo Gisondi: None declared, Luca Idolazzi: None declared, Roberta Ramonda Speakers bureau: Novartis, Celgene, Janssen, Pfizer, Abbvie, Lilly, Stefano Piaserico: None declared, Alberto Cauli: None declared, Marco Amedeo Cimmino: None declared, Veronica Tomatis: None declared, Carlo Salvarani: None declared, Rosanna Scrivo: None declared, Anna Zanetti: None declared, Greta Carrara: None declared, Carlo Alberto Scirè: None declared, Angelo Cattaneo: None declared, Antonio Marchesoni Speakers bureau: Abbvie, Pfizer, UCB, Novartis, Celgene, Eli Lilly DOI: 10.1136/annrheumdis-2020-eular.3697

\section{SAT0413 DACTYLITIS IS ASSOCIATED WITH DISEASE SEVERITY AND ULTRASOUND DEFINED EROSIVE DAMAGE IN VERY EARLY, DMARD NAÏVE PSORIATIC ARTHRITIS}

S. Dubash ${ }^{1}$, O. Alabas ${ }^{1}$, X. Michelena ${ }^{1}$, G. De Marco ${ }^{1}$, L. Garcia-Montoya ${ }^{1}$, R. Wakefield ${ }^{1}$, A. L. Tan ${ }^{1}$, P. Helliwell' ${ }^{1}$, P. Emery ${ }^{1}$, D. Mcgonagle ${ }^{1}$, H. MarzoOrtega ${ }^{1} .{ }^{1}$ NIHR LBRC, Leeds Teaching Hospitals Trust \& LIRMM, University of Leeds, Leeds, United Kingdom

Background: Dactylitis is a hallmark feature of Psoriatic arthritis (PsA) and Spondyloarthritis (SpA) defined as a uniform swelling of a digit ("sausage digit"). Dactylitis is associated with radiographic damage in chronic PsA. However, there are a paucity of data on the significance of dactylitis and its potential impact in disease burden in early PsA.

Objectives: To characterize a very early DMARD naïve PsA cohort based on clinical presence or absence of dactylitis at disease onset.

Methods: PsA subjects fulfilling the CASPAR classification criteria, were recruited into a prospective observational cohort, the Leeds Spondyloarthropathy Register for Research and Observation (SpARRO) after providing informed written consent. Clinical data including tender (TJC) and swollen joint counts (SJC) were independently assessed. Dactylitis was recorded per digit (finger or toe) as tender (hot) or non-tender (cold). Differences in baseline characteristics were evaluated using percentages to describe categorical variables and means and standard deviations for continuous variables, $p$ value of the mean/proportion difference was calculated. Ultrasound (US) examination was conducted by trained ultra-sonographers blinded to clinical details. Bone erosions were defined on US if intra-articular discontinuity was present in two perpendicular planes at any of 46 joints: wrists, MCP1-5, PIP2-5, DIP2-5, MTP1-5, knees, ankles, subtalar, talonavicular. Results: A total of 177 PsA patients were recruited. Dactylitis was seen in nearly half the cohort $[n=83(47 \%)]$. Patients with dactylitis had significantly more early morning stiffness, higher TJC and SJC, compared with non-dactylitis (Table 1). A total of 211 digits with dactylitis were recorded in 83 patients. Dactylitis of multiple digits was seen in $47 / 83(57 \%)$ patients whilst a single dactylitic digit occurred in $36 / 83(43 \%)$. Foot involvement was more prevalent $(141 / 211,67 \%)$ than hands (70/211, 33\%). "Hot" or tender dactylitis was more frequently detected (153/211, 